Nervenarzt 2021 · 92:1305-1314

https://doi.org/10.1007/s00115-021-01223-9

Angenommen: 14. Oktober 2021

Online publiziert: 25 . November 2021

(c) Der/die Autor(en) 2021

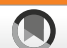

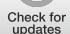

CME

Zertifizierte Fortbildung

\section{Tumortherapieassoziierte neurologische Symptome}

\author{
Mirjam Renovanz $z^{1,2} \cdot$ Johannes Rieger $^{1,2} \cdot$ Ghazaleh Tabatabai ${ }^{1,2}$ \\ ${ }^{1}$ Abteilung Neurologie mit interdisziplinärem Schwerpunkt Neuroonkologie, Universitätsklinikum \\ Tübingen, Hertie-Institut für Klinische Hirnforschung, Eberhard-Karls-Universität Tübingen, Tübingen, \\ Deutschland \\ ${ }^{2}$ Zentrum für Neuroonkologie, Comprehensive Cancer Center Tübingen-Stuttgart, Universitätsklinikum \\ Tübingen, Eberhard-Karls-Universität Tübingen, Tübingen, Deutschland
}

\section{Zusammenfassung}

Online teilnehmen unter:

www.springermedizin.de/cme

Für diese Fortbildungseinheit

werden 3 Punkte vergeben.

\section{Kontakt}

Springer Medizin Kundenservice

Tel. 08007780777

(kostenfrei in Deutschland)

E-Mail:

kundenservice@springermedizin.de

\section{Informationen}

zur Teilnahme und Zertifizierung finden Sie im CME-Fragebogen am Ende des Beitrags.

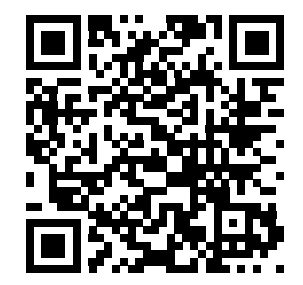

Die onkologische Behandlung ist biomarkerbasierter, molekular maßgeschneiderter und effektiver geworden. Aufbauend auf der zunehmenden Entschlüsselung zellbiologischer und molekularer Mechanismen steigt auch die Zahl zielgerichteter medikamentöser Therapien. Es steigt zudem die Zahl der Langzeitüberlebenden. Eine neuro(onko)logische Betreuung wird immer wichtiger, nicht nur wegen vermehrter direkter tumorbedingter Symptome - wie etwa der höheren Inzidenz einer Metastasierung in das Zentralnervensystem -, sondern weil im Zuge dieser modernen onkologischen systemischen Therapieformen ein breites Spektrum therapieassoziierter neurologischer Symptome auftritt, die einer sorgfältigen und raschen neurologischen/neuroonkologischen Evaluation und Therapiekonzeption bedürfen. Das Ziel dieses Artikels ist es, das Bewusstsein für die häufigsten therapieassoziierten neurologischen Symptome zu schärfen.

\section{Schlüsselwörter}

Neurotoxizität · Zentralnervensystem · Peripheres Nervensystem · Enzephalopathie · Myopathie

\section{Lernziele}

Nach der Lektüre dieses Beitrages ...

- kennen Sie die tumortherapieassoziierten neurologische Symptome des zentralen und peripheren Nervensystems,

- wissen Sie, wie je nach Schwere der Symptome systematisch klinisch und diagnostisch vorzugehen ist,

- sind Sie über das Vorgehen bei selteneren therapieassoziierten neurologischen Syndromen informiert,

- wissen Sie, welche Therapiestrategien anzuwenden sind.

QR-Code scannen \& Beitrag online lesen 


\section{Hintergrund}

Die therapieassoziierten neurologischen Symptome moderner onkologischer Therapien gehen über die langjährig bekannten chemotherapieassoziierten Polyneuropathien hinaus. Das liegt daran, dass zunehmend zielgerichtete Therapiestrategien angewendet werden. Sie greifen spezifisch an speziellen Molekülen oder Signaltransduktionswegen an, deren Bedeutung für die Krebsentstehung und -fortdauer durch grundlagenwissenschaftliche Arbeiten im Laufe der letzten Jahre aufgeklärt wurde. Viele dieser molekularen Zielstrukturen liegen auch in Kompartimenten des zentralen und/oder peripheren Nervensystems und der Muskulatur vor. Eine Modifikation dieser Moleküle und Signaltransduktionswege im Rahmen zielgerichteter Tumortherapien behandeln folglich nicht nur die Tumorerkrankung, sondern führen auch zu therapieassoziierten neurologischen Symptomen.

Zuletzt stark in den klinischen Fokus gerückt sind neurologische Komplikationen bei Checkpoint-Inhibitoren, die z. B. in der Behandlung des malignen Melanoms eingesetzt werden. Therapieassoziierte neurologische Symptome treten mit einer Inzidenz von 3,8\% bei Anti-CTLA4 („,cytotoxic T lymphocyte-associated antigen $4^{\prime \prime}$ )-Therapie (z. B. Ipilimumab), 6,1\% bei Anti-PD-1(,programmed cell death protein 1")Antikörpern (z. B. Nivolumab und Pembrolizumab) bzw. $12 \%$ bei einer Kombinationstherapie auf [1, 2]. Weitere Therapien, die im klinischen Alltag häufig verwendet werden und zu neurologischen Symptomen bzw. deren Aggravation führen können, sind z.B. Temozolomid, Procarbazin, Vincristin, Lomustin, Bevacizumab, Marizomib und "Chimeric-antigenreceptor"(CAR)-T-Zellen [3].

\section{Systematisches klinisches und diagnostisches Vorgehen}

Für den klinischen Alltag bedeutet dies, dass Neurologinnen und Neurologen zunehmend mit neurologischen Symptomen onkologischer Patientinnen und Patienten konfrontiert werden und um Mitbeurteilung und Mitbehandlung gebeten werden [1, 4]. Bei neurologischer Vorstellung eines Tumorpatienten/einer Tumorpatientin muss eine zügige und systematische Abklärung erfolgen, um zwischen tumorbedingter und therapieassoziierter oder auch anderer Ursache unterscheiden zu können (Abb. 1).

Die neurologische Anamneseerhebung und klinische Untersuchung erfolgt wie üblich, eine sehr sorgfältige Medikamentenanamnese ist für die differenzialdiagnostische Abklärung besonders essenziell, und basierend auf der neuroanatomisch-topischen Zuordnung kann die laborchemische, Liquor- und apparative Zusatzdiagnostik in die Wege geleitet werden.

Eine wichtige initial zu klärende Frage ist, ob die neurologische Symptomatik tumorbedingt ist, also Ausdruck einer Tumorprogression ist. Tumortherapieassoziierte Symptome treten üblicherweise 3 bis 12 Wochen nach dem Beginn der Therapie auf, selten sogar später [5,6]. Daher sind eine sorgfältige neurologische Ausbreitungsdiagnostik inklusive zerebraler und spinaler $\mathrm{Ma}$ gnetresonanztomographie (MRT) und Liquordiagnostik wichtige diagnostische Maßnahmen.

\section{Neurological symptoms associated with cancer} treatment

Systemic treatment in oncology has become more biomarker-based, molecularly tailored and effective. Building on increasing scientific insights into cell biological and molecular mechanisms, the number of targeted drug therapies is also increasing. There is also an increase in the number of long-term survivors. Neuro(onco)logical care is becoming increasingly more important, not only because of increased direct tumor-related symptoms, such as higher incidence of central nervous system metastases, but also because a broad spectrum of treatmentassociated neurological symptoms occur during the course of these modern oncological systemic therapies, which require careful and fast neurological/neuro-oncological evaluation and treatment. The goal of this article is to raise awareness of the most common treatmentassociated neurologic symptoms.

\section{Keywords}

Neurotoxicity - Central nervous system - Peripheral nervous system . Encephalopathy $\cdot$ Myopathy

Liegt eine Tumorprogression vor, sollten die weiteren Schritte im interdisziplinären Tumorboard diskutiert werden. Insbesondere ist dabei natürlich zu prüfen, ob eine Behandlung im Rahmen klinischer Studien oder leitliniengerecht verfügbar ist. Sollte dies nicht möglich sein, kann in spezialisierten Zentren eine erweiterte molekulare Diagnostik erfolgen, um durch das molekulare Profil mögliche zielgerichtete Therapiestrategien auswählen zu können. Diese können dann im „molekularen Tumorboard“ (MTB) priorisiert und anschließend im interdisziplinären Tumorboard im Hinblick auf die Einsetzbarkeit diskutiert werden. Je nach klinischem Zustand sind ggf. auch eine Deeskalation der Tumortherapie und eine symptomorientierte Behandlung indiziert.

Falls keine direkte tumorbedingte Ursache der neurologischen Symptome eruierbar ist, sind therapieassoziierte neurologische Symptome, ein paraneoplastisches neurologisches Syndrom oder eine andere „eigenständige“ neurologische Zweiterkrankung in Erwägung zu ziehen.

Im Folgenden liegt der Fokus dieses Artikels auf den häufigsten therapieassoziierten neurologischen Symptomen. Wir weisen darauf hin, dass sich Behandlungsstrategien in diesem Bereich fortlaufend ändern können, sodass wir empfehlen, bei entsprechender klinischer Arbeitshypothese sehr zügig die diagnostischen Wege einzuleiten - je nach Dynamik und Schwere der Symptomatik im Rahmen einer stationären Einweisung - und die Behandlung gemeinsam mit einem zertifizierten neuroonkologischen Zentrum durchzuführen.

\section{Das Wichtigste für immuntherapieassoziierte Nebenwirkungen auf einen Blick}

- Therapieassoziierte neurologische Symptome treten meist zwischen 3 und 12 Wochen nach Beginn der Therapie auf [5, 6], individuell kann dies jedoch deutlich abweichen.

- Die Einteilung erfolgt nach Common Terminology Criteria for Adverse Events (CTC AE). 


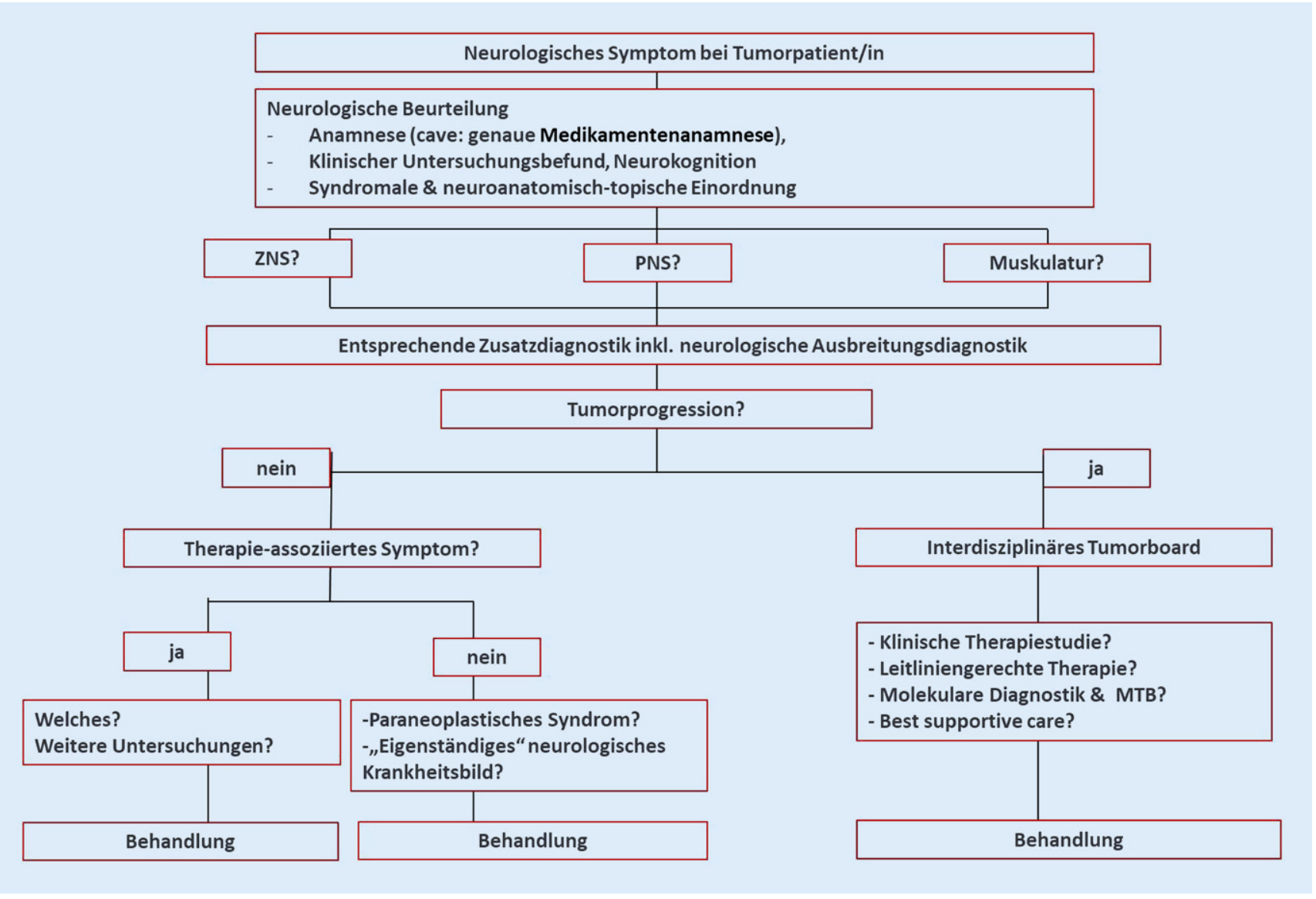

Abb. 1 ॥ Systematische Abklärung neurologischer Symptome bei TumorpatientInnen. MTB molekulares Tumorboard, PNS peripheres Nervensystem, ZNS Zentralnervensystem

- Bei der differenzialdiagnostischen Abklärung sind vor allem Tumorprogression, epileptische Anfälle, Infektionen und metabolische Ursachen in Betracht zu ziehen.

- Bei milden neurologischen Symptomen (CTC-AE-Grad I, mild) sollte die Immuntherapie pausiert werden, bis alle weiteren möglichen Ursachen abgeklärt wurden.

- Prednisolon 0,5-1 mg/kg Körpergewicht (KG) oral wird für die Behandlung moderater Symptome empfohlen (CTC-AE-Grad II).

- Bei mittelschweren Symptomen (CTC-AE-Grad III) sollte Prednisolon 1-2 mg/kgKG i.v. verabreicht werden, bei schwer (CTCAE-Grad IV) betroffenen PatientInnen kann auch eine höher dosierte Behandlung mit Methylprednisolon mit $500-1000 \mathrm{mg}$ über 3 bis 5 Tage gewählt werden.

- Intravenöse Immunglobuline (IVIG) und Plasmapherese sind vor allem bei myasthenem Syndrom, Guillain-Barré-Syndrom (GBS) und transverser Myelitis indiziert.

- Die ZNS(Zentralnervensystem)-Toxizität nach Behandlung mit CAR-T-Zellen muss sehr genau diagnostisch eingeordnet werden, ggf. ist eine Therapie mit Tocilizumab, einem gegen den Interleukin-6-Rezeptor gerichteten Antikörper, einzusetzen [3].
> Merke

- Es kommt auf eine zügige Diagnostik und rasche Einleitung der Therapie an.

- In der Regel gilt für die Behandlung immuntherapieassoziierter neurologischer Nebenwirkungen "steroide first".

- Eine sehr enge und hochfrequente interdisziplinäre Abstimmung ist entscheidend (z. B. mit der primär behandelnden onkologischen Disziplin sowie ggf. weiteren Disziplinen bei Multiorganbeteiligung).

\section{Peripheres Nervensystem und Muskulatur}

Das periphere Nervensystem und die Muskulatur sind gerade bei den immuntherapieassoziierten neurologischen Nebenwirkungen häufiger betroffen als das zentrale Nervensystem.

\section{(Poly-)Neuro- und Radikulopathie}

Eine Vielzahl zielgerichteter Therapien kann zu peripheren Neuropathien führen. Für die klassischen Zytostatika wie Vincristin oder Taxane oder Platinderivate ist dies schon lange bekannt, unter den modernen Therapien gilt dies z. B. für Proteasominhibitoren, PD-1-Antikörper und PD-L1 („programmed death-ligand 1“)Antikörper (z. B. Durvalumab, Atezolizumab und Avelumab), weitere immunmodulatorische Substanzen sowie Antikörper-Wirkstoff- 
Konjugate [7]. Die Symptome bleiben oft auch nach Abschluss der Behandlung bestehen und beeinträchtigen die langfristige Funktion und Lebensqualität [7].

Für den klinischen Alltag ist zu beachten, dass eine vorbestehende diabetische Neuropathie natürlich die Effekte nicht nur maskieren, sondern auch verstärken kann. Es scheint so zu sein, dass tumorassoziierte Neuropathien bei diabetischen PatientInnen länger anhalten. In einer rezenten Studie persistierte die periphere Neuropathie bei einem höheren Anteil der diabetischen PatientInnen (im Vergleich zu nichtdiabetischen PatientInnen) bis zu 2 Jahre nach der Behandlung. Es wird sicher wichtig sein, in künftigen Studien das Zusammenspiel diabetischer Neuropathien und tumorassoziierter Neuropathien weiter herauszuarbeiten. Wichtige Parameter könnten hierbei z. B. die Form und Dauer des Diabetes sowie die antidiabetische Therapie sein [8].

Bei der tumortherapieassoziierten Polyneuropathie sind meist Dosisreduktion, Therapiepause bis hin zu Therapieabbruch die einzige Strategie zur Verhinderung schwerer tumortherapieassoziierter Neuropathien, was im klinischen Alltag oft herausfordernd ist: Wie soll ein Gleichgewicht gefunden werden zwischen maximaler Behandlungsexposition und minimalen langfristigen Nebenwirkungen? Es wäre daher empfehlenswert, bereits vor dem Start der onkologischen Therapie eine neurologische und neurographische Untersuchung als Ausgangsbefund durchzuführen und diese u.U. auch im Verlauf regelmäßig zu wiederholen. Ein neu- rologisches Monitoring würde es sicher leichter ermöglichen, die Behandlung durch eine engmaschigere Überwachung von RisikopatientInnen zu personalisieren [9]. Hierzu ist eine enge interdisziplinäre Zusammenarbeit erforderlich. An dieser Stelle sei der Vollständigkeit halber darauf hingewiesen, dass es noch keine evidenzbasierte Prophylaxe für eine tumortherapieassoziierte Neuropathie gibt. Die bisherige Literatur zu Vitamin E, das gehäuft eingesetzt wurde, zeigt keine überzeugenden Belege hierfür: Ergebnisse doppelblinder randomisierter klinischer Studien deuten jedenfalls darauf hin, dass Vitamin E die Inzidenz tumortherapieassoziierter Neuropathien nicht signifikant reduzierte. Zudem gab es auch keinen signifikanten Unterschied in der Häufigkeit schwerer tumortherapieassoziierter Neuropathien zwischen beiden Gruppen. Die routinemäßige prophylaktische Einnahme von Vitamin E empfehlen wir daher aktuell nicht, bis entsprechend Daten aus weiteren doppelblind randomisierten klinischen Studien, die die präventive Wirkung von Vitamin E untersuchen, vorliegen [10].

In Tab. 1 ist am Beispiel der immuntherapieassoziierten Neuropathie das stufenweise diagnostische und therapeutische Vorgehen dargestellt.

\begin{tabular}{|c|c|c|}
\hline $\begin{array}{l}\text { Toxizitätsgrad nach } \\
\text { CTC-AE-Kriterien }\end{array}$ & Diagnostik und Verlaufskontrolle & Behandlung \\
\hline $\begin{array}{l}\text { Grad l: asymptoma- } \\
\text { tischer Befund, milde } \\
\text { Symptome, keine } \\
\text { Funktionseinschrän- } \\
\text { kung }\end{array}$ & $\begin{array}{l}\text { Klinisch-neurologische Untersuchung, PNP-Labor } \\
\text { (Vitamin B12, Folsäure, Diabetes und HIV-Screening), } \\
\text { CMRT, sMRT oder Plexusdarstellung mit und ohne } \\
\text { Kontrastmittelgabe, elektrophysiologische Diagnostik }\end{array}$ & $\begin{array}{l}\text { Fortführung der Immuntherapie möglich, ggf. Dosisreduktion oder } \\
\text { Pause }\end{array}$ \\
\hline \multirow{4}{*}{$\begin{array}{l}\text { Grad II: mittelgradige } \\
\text { Symptome, leichte } \\
\text { Beeinträchtigung im } \\
\text { Alltag, Hirnnervenbe- } \\
\text { teiligung }\end{array}$} & \multirow{2}{*}{$\begin{array}{l}\text { Wie bei Grad I } \\
\text { zusätzlich Lumbalpunktion, Lungenfunktionsprüfung }\end{array}$} & Immuntherapie pausieren \\
\hline & & Methylprednisolon 0,5-1 mg/kg Körpergewicht/Tag i.v. über 5 Tage \\
\hline & $\begin{array}{l}\text { 4-wöchige neurologisch/neuroonkologische Ver- } \\
\text { laufskontrolle bis zum vollständigen Abklingen bzw. } \\
\text { möglichst Besserung Grad I }\end{array}$ & $\begin{array}{l}\rightarrow \text { Nach Therapieansprechen: dosisäquivalente orale Prednisolon- } \\
\text { gabe und schrittweise Ausschleichen über 4-6 Wochen }\end{array}$ \\
\hline & $\begin{array}{l}\text { CAVE: bei Rebound-Phänomen unter Reduktion bzw. } \\
\text { Verschlechterung nach Therapieansprechen, ggf. } \\
\text { Eskalation erwägen }\end{array}$ & $\rightarrow$ Bei fehlendem Therapieansprechen: wie Grad III-IV behandeln \\
\hline \multirow{6}{*}{$\begin{array}{l}\text { Grad III-IV: schwere } \\
\text { Symptome, potenziell } \\
\text { lebensgefährliche } \\
\text { Nebenwirkungen, z. B. } \\
\text { Dyspnoe }\end{array}$} & Wie bei Grad I und II & \multirow{2}{*}{$\begin{array}{l}\text { Immuntherapie absetzen, stationäre Aufnahme } \\
\text { (Je nach Manifestation und Dringlichkeit, strenge Nutzen-Risiko- } \\
\text { Abwägung bei der Reevaluation - nur im Falle vollständiger Erho- } \\
\text { lung neurologischer Erkrankung) }\end{array}$} \\
\hline & $\begin{array}{l}\text { Neuro(onko)logische stationäre Behandlung, 4-wö- } \\
\text { chige Verlaufskontrolle in neuroonkologischer Ambu- } \\
\text { lanz bis zum vollständigen Abklingen bzw. möglichst } \\
\text { Besserung Grad I }\end{array}$ & \\
\hline & \multirow{4}{*}{$\begin{array}{l}\text { CAVE: bei Rebound-Phänomen unter Reduktion bzw. } \\
\text { Verschlechterung nach Therapieansprechen ggf. } \\
\text { Eskalation erwägen }\end{array}$} & Methylprednisolon 2 mg/kg Körpergewicht / Tag i.v. über 5 Tage \\
\hline & & $\begin{array}{l}\rightarrow \text { Nach Therapieansprechen: } 1 \mathrm{mg} / \mathrm{kg} \text { Körpergewicht /Tag orale } \\
\text { Prednisolongabe und schrittweise Ausschleichen über 4-6 Wochen }\end{array}$ \\
\hline & & $\begin{array}{l}\rightarrow \text { Bei fehlendem Ansprechen bzw. Rebound-Phänomen unter Ste- } \\
\text { roidreduktion: individuelles Erwägen weiterer Immunmodulation } \\
\text { (z. B. IVIG, Methotrexat, Infliximab, Tocilizumab) je nach neurologi- } \\
\text { scher Manifestation, Organbeteiligung und onkologischem Staging }\end{array}$ \\
\hline & & $\begin{array}{l}\rightarrow \text { Bei fehlendem Ansprechen Anti-CD-20-Antikörper erwägen [13, } \\
\text { 14] }\end{array}$ \\
\hline
\end{tabular}


Myopathien, Myositiden, myasthene Syndrome

Patientinnen und Patienten mit Krebserkrankungen schildern häufig subjektive Beschwerden im Bereich der Muskulatur. Diese können auf tumorbedingte Kachexie und/oder auf längerfristige Steroideinnahme zurückführbar sein, daher gilt es stets, bei diesem Beschwerdekomplex eine sorgfältige Ernährungsberatung zu veranlassen und die Steroiddosis zu reevaluieren, zudem nach Fatigue und körperlicher Aktivität zu fragen. Die therapieassoziierten Myopathien, die unter Immuncheckpoint-Inhibitoren (z. B. PD-1-Antikörper oder CTLA4-Antikörper) oder unter dem Proteinkinaseinhibitor Imatinib oder dem MEK1/2(„mitogen activated protein kinase 1 and 2")-Inhibitor Selumetinib auftreten, können allerdings auch die Rumpfmuskulatur betreffen [10], daher ist eine sorgfältige klinische Evaluation von entscheidender Bedeutung für eine zielführende (Differenzial-)Diagnose.

Das diagnostische Vorgehen entspricht im Wesentlichen den Leitlinien der Deutschen Gesellschaft für Neurologie. Eine Muskelbiopsie kann bei unklarer Faktenlage helfen, tumorbedingte vs. therapieassoziierte vs. andere Myopathien zu differenzieren. Es ist zudem wichtig, eine kardiologische Abklärung nicht zu vergessen, da gerade Immuntherapien zu Myokarditiden und Kardiomysositiden führen können $[15,16]$.

Therapieassoziierte myasthene Syndrome, die auch bereits durchaus frühzeitig als therapieassoziierte neurologische Symptome, insbesondere unter Immuntherapien auftreten können, ähneln den „regulären“ Erscheinungsformen dieser Erkrankungen. Entsprechend sind diagnostisches und therapeutisches Vorgehen ähnlich und gemäß den Leitlinien der Deutschen Gesellschaft für Neurologie vorzunehmen (siehe auch Tab. 2).

\section{Zentrales Nervensystem}

\section{Hypophysitis}

Die Hypophysitis gehört zu den prominenten und häufigsten therapieassoziierten neurologischen Symptomen unter Immuncheckpoint-Inhibition, hier insbesondere unter dem CTLA4-Inhibitor

\begin{tabular}{|c|c|c|}
\hline $\begin{array}{l}\text { Syndrom (Ver- } \\
\text { dachtsdiagnose) }\end{array}$ & Diagnostik und Verlaufskontrolle & Behandlung \\
\hline \multirow{2}{*}{$\begin{array}{l}\text { Guillain-Barré- } \\
\text { Syndrom }\end{array}$} & Umgehende Diagnostik und rasche Therapieeinleitung! & \multirow{2}{*}{$\begin{array}{l}\text { Beim Verdacht auf therapieasso- } \\
\text { ziiertes GBS: Methylprednisolon } \\
1-2 \mathrm{mg} / \mathrm{kg} \text { Körpergewicht } \\
\text { Frühzeitig IVIG erwägen } \\
\text { Intensivüberwachung }\end{array}$} \\
\hline & $\begin{array}{l}\text { Elektrophysiologische Diagnostik } \\
\text { Lumbalpunktion (erhöhtes Protein, normale Zellzahl, Antikörpertestung für GBS-Vari- } \\
\text { anten) } \\
\text { Lungenfunktion mit Vitalkapazität }\end{array}$ & \\
\hline \multirow{4}{*}{$\begin{array}{l}\text { Myasthenia-gra- } \\
\text { vis/myasthene- } \\
\text { Syndrome }\end{array}$} & $\begin{array}{l}\text { Neurologische Untersuchung, besonderer Fokus auf Okulomotorik und proximale } \\
\text { Muskelgruppen }\end{array}$ & $\begin{array}{l}\text { Methylprednisolon } 1-2 \mathrm{mg} / \mathrm{kg} \text { Kör- } \\
\text { pergewicht i.v. über } 5 \text { Tage }\end{array}$ \\
\hline & Tensilontest & Pyridostigmin, $30 \mathrm{mg}$ initiale Dosis \\
\hline & \multirow[t]{2}{*}{ Elektrophysiologische Diagnostik (repetitive Stimulation) } & $\begin{array}{l}\text { Bei Nichtbesserung: Plasmapherese } \\
\text { oder IVIG-Gabe }\end{array}$ \\
\hline & & $\begin{array}{l}\text { Weitere Immunsuppression z. B. mit } \\
\text { Azathioprin oder Cyclophosphamid }\end{array}$ \\
\hline \multirow[t]{4}{*}{$\begin{array}{l}\text { Aseptische } \mathrm{Me-} \\
\text { ningitis und/oder } \\
\text { Enzephalitis }\end{array}$} & $\begin{array}{l}\text { Ausschluss Hirndruck, cMRT und sMRT mit und ohne Kontrastmittelgabe vor Lumbal- } \\
\text { punktion (Eröffnungsdruck, Zellzahl und Basisdiagnostik inkl. IgG-Index, Zytologie/ } \\
\text { Neuropathologie, PCR für HSV, VZV, Mikrobiologie: Borrelien) } \\
\text { Blutkulturen }\end{array}$ & \multirow{4}{*}{$\begin{array}{l}\text { Methylprednisolon } 2 \mathrm{mg} / \mathrm{kg} \text { Körper- } \\
\text { gewicht oder bei schwer betroffenem } \\
\text { Patientlnnen } 500-1000 \mathrm{mg} \text { über } \\
5 \text { Tage nach Erregerdiagnostik, bei } \\
\text { noch ausstehenden virologischen } \\
\text { und mikrobiologischen Befunden } \\
\text { zusätzlich i.v. Aciclovir und Antibiose } \\
\text { nach DGN-Leitlinie }\end{array}$} \\
\hline & EEG & \\
\hline & $\begin{array}{l}\text { Beim Verdacht auf Enzephalitis metabolische Ursachen evaluieren (z. B. Kalzium, Am- } \\
\text { moniak, TSH) }\end{array}$ & \\
\hline & $\begin{array}{l}\text { Seltene Antikörper (NMDA-Rezeptor-Antikörper oder paraneoplastische, onkoneurale } \\
\text { Antikörper wie z. B. Anti-Hu, Anti-CASPR2, Anti-NMDA-Rezeptor) berücksichtigen [2, 17, } \\
\text { 18] }\end{array}$ & \\
\hline \multirow{3}{*}{$\begin{array}{l}\text { Transverse Myeli- } \\
\text { tis }\end{array}$} & cMRT und sMRT mit und ohne Kontrastmittelgabe vor Lumbalpunktion & \multirow{3}{*}{$\begin{array}{l}\text { Methylprednisolon 1-2 mg/kg Kör- } \\
\text { pergewicht } \\
\text { Frühzeitig IVIG und Plasmapherese } \\
\text { erwägen }\end{array}$} \\
\hline & $\begin{array}{l}\text { Serum-Vitamin B12, HIV, Syphilis, ANA, Anti-Ro- und, Anti-La-Antikörper, Anti-Aquapo- } \\
\text { rin4-IgG, TSH }\end{array}$ & \\
\hline & Elektrophysiologische Diagnostik & \\
\hline \multirow[t]{4}{*}{ Myopathie } & Labor: CK, CK-MB, Troponin, Transaminasen & \multirow{4}{*}{$\begin{array}{l}\text { Methylprednisolon 1-2 mg/kg Kör- } \\
\text { pergewicht }\end{array}$} \\
\hline & Elektrophysiologie (EMG, NLG, repetitive Stimulation) & \\
\hline & EKG, Herzechokardiogramm & \\
\hline & Muskelbi & \\
\hline
\end{tabular}

CK Kreatinkinase, cMRT kranielle Magnetresonanztomographie, DGN Deutsche Gesellschaft für Neurologie, EKG Elektrokardiographie, EMG Elektromyographie, GBS Guillain-Barré-Syndrom, HIV humanes Immundefizienzvirus, IVIG intravenöse Immunglobuline, HSV Herpes-simplex-Virus, IgG immunglobulin G, NLG Nervenleitungsgeschwindigkeit, NMDA N-Methyl-D-Aspartat, $P C R$ „polymerase chain reaction“, sMRT spinale Magnetresonanztomographie, TSH thyreoideastimulierendes Hormon, VZV Varizella-Zoster-Virus 
Ipilimumab, deutlich seltener unter PD-1-/PD-L1-Blokade. Die Hypophysitis tritt bei Ipilimumabbehandlung bei knapp 10\% der behandelten Patientinnen und Patienten bereits innerhalb der ersten 6 bis 8 Wochen auf [19]. Die Diagnose kann dadurch erschwert werden, dass die Beschwerden der PatientInnen sehr unspezifisch sein können. Wir empfehlen daher, die Differenzialdiagnose einer Hypophysitis bei stattgehabter Immuncheckpoint-Inhibition zu konsiderieren und sorgfältig abzuklären, dies zudem auch bei unklaren therapieassoziierten neurologischen Symptomen unter anderen Tumortherapien.

\section{Enzephalopathien}

Enzephalopathien können durchaus rasch nach dem Therapiestart mit klassischen zytotoxischen Zytostatika auftreten und somit in der Regel unschwer als therapieassoziiert erkannt werden, z. B. induziert durch das Alkylanz Ifosfamid bzw. durch intrathekale Applikation des Folsäureantagonisten Methotrexat.

Therapieassoziierte Enzephalopathien im Zusammenhang mit medikamentösen Systemtherapien oder auch intrathekalen Therapien, die nach einer Ganzhirnbestrahlung auftreten, haben häufig schwere und irreversible Verläufe [20].

Auch strahlentherapieinduzierte Effekte unter Checkpoint-Inhibition sollten in Betracht gezogen werden: Hierbei kann es vermehrt zum sog. abskopalen Effekt kommen. Dies bedeutet, dass z. B. nach einer extrakraniellen Bestrahlung ein intrakranielles Ansprechen durch eine radiotherapieinduzierte Immunmodulation beobachtet werden kann [21].

Auch die modernen zielgerichteten onkologischen Therapien, z. B. Proteasominhibitoren (z. B. Bortezomib), können akute Enzephalopathien verursachen [22], ebenso Immuncheckpoint-Inhibitoren und CAR-T-Zellen. Der Entstehungsmechanismus dieser verschiedenen Formen therapieassoziierter Enzephalopathien ist jeweils verschieden. Wichtig und allen Formen gemeinsam ist, dass eine Abgrenzung zu erregerbedingten (vor allem viralen) Enzephalitiden rasch erfolgen muss. Wir empfehlen bei einer entzündlichen Liquorkonstellation bis zum negativen HSV(Herpessimplex-Virus)- und VZV(Varizella-Zoster-Virus)-PCR(„polymerase chain reaction")-Test eine Aciclovirtherapie zu initiieren (siehe auch Tab. 2).

\section{Aseptische Meningitis}

Zwar ist die Diagnose einer aseptischen Meningitis dadurch erschwert, dass es keine beweisenden diagnostischen Befunde gibt. Umso wichtiger ist es, diese bei unklarer Liquorpleozytose und fehlendem Erregernachweis stets differenzialdiagnostisch zu erwägen. Erhöhte Zytokinwerte im Liquor können hilfreich sein, ebenso eine bildmorphologische Kontrastmittelanhebung der Meningen. Dokumentierte Beobachtungen aseptischer Meningitiden gibt es unter Immuncheckpoint-Therapie [23] und auch unter dem Multikinaseinhibitor Regorafenib [24]. In der Regel ist dieses Krankheitsbild steroidresponsiv, sodass eine Prednisolontherapie bereits zügig zu einer Verbesserung führen kann (siehe auch Tab. 2).

\section{Therapiestrategien}

Die nationalen und europäischen Fachgesellschaften aktualisieren fortlaufend die Empfehlungen für die Behandlungsstrategien therapieassoziierter neurologischer Symptome. Dies trifft sicherlich am weitesten für die Immuncheckpoint-Inhibitoren und für die CAR-T-Zellen zu. Wir empfehlen daher die alerte und zügige neurologische Evaluation und Differenzialdiagnostik und die Zusammenarbeit mit einem zertifizierten neuroonkologischen Zentrum. Zu betonen ist, dass einige der therapieassoziierten neurologischen Symptome, insbesondere unter den Immuntherapien, sehr rasch lebensbedrohlich werden können. Da sie aber meistens gleichzeitig auch therapierbar sind, obliegt den erstevaluierenden ärztlichen Kolleginnen und Kollegen eine sehr hohe Verantwortung, die Situation richtig einzuordnen und die entsprechenden Schritte zügig einzuleiten. Konzeptionell dargelegt bestehen die wichtigsten Maßnahmen aus dem Absetzen der auslösenden Medikation, einer engmaschigen klinischen Evaluation, bei Persistenz oder Verschlechterung müssen dann je nach Pathomechanismus eine Prednisolontherapie, intravenöse Immunglobuline, Azathioprin, Tocilizumab oder auch eine Plasmapherese konsideriert werden.

Die Therapiedauer ist abhängig von der klinischen Symptomatik und Entwicklung der jeweils im Vordergrund stehenden laborchemischen, liquordiagnostischen und/oder bildgebenden Diagnostik (siehe Tab. 1 und 2).

\section{Fazit für die Praxis}

- In der Behandlung von Tumorerkrankungen ist die Verlängerung von progressionsfreiem und Gesamtüberleben ein vorrangiges Ziel. Gleichermaßen wichtig ist die berichtete Lebensqualität, denn therapieassoziierte neurologische Symptome können nicht nur die Therapiedurchführung, sondern insbesondere die Lebensqualität der PatientInnen stark beeinträchtigen.

- Es wird künftig wichtig sein, Biomarker zu identifizieren, die helfen, das Auftreten therapieassoziierter (neurologischer) Symptome vorherzusehen und eine entsprechende Stratifizierung bei der Therapieplanung vorzunehmen.

- Zudem formulierte aktuell eine Expertengruppe für bestimmte Tumorentitäten und ausgewählte Therapieformen die Einführung eines neuen klinischen Endpunkts, das "severe toxicity-free survival", d.h. der zeitliche Abstand zwischen Therapiestart und Auftreten einer schweren therapieassoziierten Symptomatik. Dieser Endpunkt gibt eine wichtige Richtung für die Weiterentwicklung onkologischer Therapien vor.

\section{Korrespondenzadresse}

\section{Prof. Dr. Dr. Ghazaleh Tabatabai}

Abteilung Neurologie mit interdisziplinärem Schwerpunkt Neuroonkologie, Universitätsklinikum Tübingen, Hertie-Institut für Klinische Hirnforschung, Eberhard-Karls-Universität Tübingen

Tübingen, Deutschland

ghazaleh.tabatabai@uni-tuebingen.de 


\section{Einhaltung ethischer Richtlinien}

Interessenkonflikt. Gemäß den Richtlinien des Springer Medizin Verlags werden Autoren und Wissenschaftliche Leitung im Rahmen der Manuskripterstellung und Manuskriptfreigabe aufgefordert, eine vollständige Erklärung zu ihren finanziellen und nichtfinanziellen Interessen abzugeben.

Autoren. M. Renovanz: A. Finanzielle Interessen: GLIOPT Studie (Innovationsfond GBA Versorgungsforschung). - B. Nichtfinanzielle Interessen: Fachärztin für Neurochirurgie, Abteilung Neurologie mit interdisz. Schwerpunkt Neuroonkologie und Klinik für Neurochirurgie Uniklinikum Tübingen. J. Rieger: A. Finanzielle Interessen J. Rieger gibt an, dass kein finanzieller Interessenkonflikt besteht. - B. Nichtfinanzielle Interessen: Oberarzt Neurologie, Abteilung Neurologie mit interdisz. Schwerpunkt Neuroonkologie und Klinik für Neurochirurgie Uniklinikum Tübingen. G. Tabatabai: A. Finanzielle Interessen: Forschungsförderung zur persönlichen Verfügung: Roche Diagnostics: Forschungsförderung für die Grundlagenforschung an die Institution zur Verwendung im Labor. - Referentenhonorar oder Kostenerstattung als passiver Teilnehmer: Kostenerstattung für Kongressbesuch: Novocure, Medac, BMS. - Bezahlter Berater/interner Schulungsreferent/Gehaltsempfänger o. ä.: Beratung: AbbVie, Bayer, BMS, Schulungsreferent: Medac, Novocure. - B. Nichtfinanzielle Interessen: Ärztliche Direktorin, Abteilung Neurologie mit interdisziplinärem Schwerpunkt Neuroonkologie, Universitätsklinikum Tübingen, Eberhard Karls Universität Tübingen | Mitgliedschaften: American Society of Oncology, European Asociation of Neuro-Oncology, European Organisation for Research and Treatment of Cancer, Schweizerische Gesellschaft für Klinische Neurophysiologie, Deutsche Gesellschaft für Neurologie, Deutsche Krebsgesellschaft, Neurowissenschaftliche Gesellschaft.

Wissenschaftliche Leitung. Die vollständige Erklärung zum Interessenkonflikt der Wissenschaftlichen Leitung finden Sie am Kurs der zertifizierten Fortbildung auf www. springermedizin.de/cme.

Der Verlag erklärt, dass für die Publikation dieser CME-Fortbildung keine Sponsorengelder an den Verlag fließen.

Für diesen Beitrag wurden von den Autoren keine Studien an Menschen oder Tieren durchgeführt. Für die aufgeführten Studien gelten die jeweils dort angegebenen ethischen Richtlinien.

Open Access. Dieser Artikel wird unter der Creative Commons Namensnennung 4.0 International Lizenz veröffentlicht, welche die Nutzung, Vervielfältigung, Bearbeitung Verbreitung und Wiedergabe in jeglichem Medium und Format erlaubt, sofern Sie den/die ursprünglichen Autor(en) und die Quelle ordnungsgemäßnennen, einen Link zur Creative Commons Lizenz beifügen und angeben, ob Änderungen vorgenommen wurden.

Die in diesem Artikel enthaltenen Bilder und sonstiges Drittmaterial unterliegen ebenfalls der genannten Creative Commons Lizenz, sofern sich aus der Abbildungslegende nichts anderes ergibt. Sofern das betreffende Material nicht unter der genannten Creative Commons Lizenz steht und die betreffende Handlung nicht nach gesetzlichen Vorschriften erlaubt ist, ist für die oben aufgeführten Weiterverwendungen des Materials die Einwilligung des jeweiligen Rechteinhabers einzuholen.

Weitere Details zur Lizenz entnehmen Sie bitte der Lizenzinformation auf http:// creativecommons.org/licenses/by/4.0/deed.de.

\section{Literatur}

1. Mildenberger I, Eisele P, Hofheinz R, Platten M (2019) Treatment-associated neurological symptoms of cancer patients and their treatment. Nervenarzt 90:587-593

2. Cuzzubbo S, Javeri F, Tissier M, Roumi A, Barlog C, Doridam J, Lebbe C, Belin C, Ursu R, Carpentier AF (2017) Neurological adverse events associated with immune checkpoint inhibitors: review of the literature. Eur J Cancer 73:1-8. https://doi.org/ 10.1016/j.ejca.2016.12.001

3. Rubin DB, Vaitkevicius H (2021) Neurological complications of cancer immunotherapy (CAR T cells). J Neurol Sci. https://doi.org/10.1016/j.jns.2021. 117405

4. Wick W, Hertenstein A, Platten M (2016) Neurological sequelae of cancer immunotherapies and targeted therapies. Lancet Oncol 17:e529-e541

5. Haanen JBAG, Carbonnel F, Robert C, Kerr KM, Peters S, Larkin J, Jordan K, ESMO Guidelines Committee (2017) Management of toxicities from immunotherapy:
ESMO Clinical Practice Guidelines for diagnosis, treatment and follow-up. Ann Oncol 28(Supplement 4):i119. https://doi.org/10.1093/annonc/mdx225

6. Hottinger AF (2016) Neurologic complications of immune checkpoint inhibitors. Curr Opin Neurol 29(6):806-812. https://doi.org/10.1097/WCO. 0000000000000391

7. Li T, Mizrahi D, Goldstein D, Kiernan MC, Park SB (2021) Chemotherapy and peripheral neuropathy. Neurol Sci 42(10):4109-4121. https://doi.org/10.1007/ s10072-021-05576-6

8. Sempere-Bigorra M, Julian-Rochina I, Cauli O (2021) Chemotherapy-induced neuropathy and diabetes: a scoping review. Curr Oncol 28:3124-3138

9. Velasco R, Alemany M, Villagran M, Argyriou AA (2021) Predictive biomarkers of Oxaliplatin-induced peripheral neurotoxicity. J Pers Med 11(7):669. https://doi. org/10.3390/jpm11070669

10. Chen J, Shan H, Yang W, Zhang J, Dai H, Ye Z (2021) Vitamin E for the prevention of chemotherapy-induced peripheral neuropathy: a meta-analysis. Front Pharmacol 12:684550

11. Guidon AC, Burton LB, Chwalisz BK et al (2021) Consensus disease definitions for neurologic immune-related adverse events of immune checkpoint inhibitors. JImmunother Cancer 9:e2890. https://doi.org/10.1136/jitc-2021-002890

12. Wesley SF, Haggiagi A, Thakur KT et al (2021) Neurological immunotoxicity from cancer treatment. Int J Mol Sci 22:6716. https://doi.org/10.3390/ijms22136716

13. Nowosielski M, Di Pauli F, Iglseder S, Wagner M, Hoellweger N, Nguyen VA, Gruber J Stockhammer G (2020) Encephalomyeloneuritis and arthritis after treatment with immune checkpoint inhibitors. Neurol Neuroimmunol Neuroinflamm 7(4):e773. https://doi.org/10.1212/NXI.0000000000000773

14. Vogrig A, Muñiz-Castrillo S, Joubert B, Picard G, Rogemond V, Marchal C, Chiappa AM, Chanson E, Skowron F, Leblanc A, Ducray F, Honnorat J (2020) Central nervous system complications associated with immune checkpoint inhibitors. J Neurol Neurosurg Psychiatry 91(7):772-778. https://doi.org/10.1136/jnnp2020-323055

15. Pasnoor M, Barohn RJ, Dimachkie MM (2018) Toxic myopathies. Curr Opin Neurol 31:575-582

16. Matzen E, Bartels LE, Logstrup B, Horskaer S, Stilling C, Donskov F (2021) Immune checkpoint inhibitor-induced myocarditis in cancer patients: a case report and review of reported cases. Cardiooncology 7:27

17. Williams TJ, Benavides DR, Patrice KA, Dalmau JO, de Ávila AL, Le DT, Lipson EJ, Probasco JC, Mowry EM (2016) Association of autoimmune encephalitis with combined immune checkpoint inhibitor treatment for metastatic cancer. JAMA Neurol 73(8):928-933. https://doi.org/10.1001/jamaneurol.2016.1399

18. Pan PC, Haggiagi A (2019) Neurologic immune-related adverse events associated with immune checkpoint inhibition. Curr Oncol Rep 21(12):108. https://doi.org/ 10.1007/s11912-019-0859-2

19. Weber JS, Kahler KC, Hauschild A (2012) Management of immune-related adverse events and kinetics of response with ipilimumab. JClin Oncol 30:2691-2697

20. Sioka C, Kyritsis AP (2009) Central and peripheral nervous system toxicity of common chemotherapeutic agents. Cancer Chemother Pharmacol 63:761-767

21. Hotta T, Okuno T, Nakao M, Amano Y, Isobe T, Tsubata Y (2021) Reproducible abscopal effect in a patient with lung cancer who underwent whole-brain irradiation and atezolizumab administration. Thorac Cancer 12(6):985-988. https://doi.org/10.1111/1759-7714.13875

22. Oshikawa G, Kojima A, Doki N, Kobayashi T, Kakihana K, Tsuda H et al (2013) Bortezomib-induced posterior reversible encephalopathy syndrome in a patient with newly diagnosed multiple myeloma. Intern Med 52:111-114

23. Roth P, Winklhofer S, Muller AMS, Dummer R, Mair MJ, Gramatzki D et al (2021) Neurological complications of cancer immunotherapy. Cancer Treat Rev 97:102189

24. Gepfner-Tuma I, Hirsch S, Schittenhelm J, Tzaridis T, Ziemann U, Tatagiba M et al (2019) Corticosteroid-responsive aseptic meningitis during regorafenib treatment. Neurooncol Pract 6:508-509 
? Eine tumortherapieassoziierte Polyneuropathie...

○ wird nicht durch Checkpoint-Inhibitoren verursacht.

- tritt nur unter Vincristintherapie auf.

O sistiert unmittelbar nach Abbruch der Therapie.

O kann durch frühzeitige Vitamin-E-Gabe verhindert werden.

O kann bei vorbestehender diabetischer Neuropathie verstärkt werden.

\section{Bei systematischer klinischer und} diagnostischer Vorgehensweise bei neurologischen Beschwerden von TumorpatientInnen ist folgende Maßnahme nicht ausreichend:

O Neurologische Untersuchung

- Laborchemische Untersuchung inklusive Zytokine

O Natives kranielles Computertomogramm

O Liquordiagnostik

O Medikamentenanamnese

Welche Fachdisziplin sollte bei PatientInnen mit Tumorerkrankungen mit Beschwerden im Bereich der Muskulatur konsultiert werden?

O Orthopädie wegen Myegelosen

○ Gastroenterologie wegen Gastroparese

○ Hämatologie wegen Osteomyelitis

○ Kardiologie wegen möglicher Myokarditis

○ Urologie wegen Blasenmuskelstörung
Welche der folgenden Tumortherapien führt am häufigsten zu neurologischen Symptomen im peripheren Nervensystem?

O CTLA4 (" "cytotoxic T lymphocyte-associated antigen 4")-PD1 („programmed cell death protein 1")/PDL1 („,programmed death-ligand 1")-Antikörper (Ipilimumab, Nivolumab, Pembrolizumab, Atezolizumab)

O CDK (" cyclin-dependent kinase")-4/6-Inhibitoren (Palbociclib, Abemaciclib, Ribociclib)

O EGFR („epidermal growth factor receptor")Inhibitoren (Afatinib, Neratinib)

O VEGF („vascular endothelial growth factor")-Antikörper (Bevacizumab)

O FGFR ("fibroblast growth factor receptor")Inhibitoren (Erdafitinib, Pemigatinib)

? Wie gehen Sie beim Verdacht auf eine Tumorprogression in das Zentralnervensystem als Ursache einer neurologischen Symptomatik einer Tumorpatientin/eines Tumorpatienten vor?

○ Primär Immunglobuline i.v.

○ Deeskalation der Therapie, da infauste Situation

O Interdisziplinäre Diskussion im Tumorboard

O Ganzhirnbestrahlung planen

○ Umgehend hochdosiert Steroide geben

Ein 65-jähriger Patient stellt sich montags in Ihrer Praxis mit neuen, über das Wochenende aufgetretenen Paresen der unteren Extremitäten Er berichtet von allgemeinem Unwohlsein, Müdigkeit und Abgeschlagenheit. Vor allem beim Aufstehen aus sitzender Position und beim Treppensteigen verspüre er eine deutliche Schwäche der Beine. In der Medikamentenanamnese erfahren Sie, dass er wegen eines malignen Melanoms mit dem AntiPD1(„programmed cell death protein 1")-Antikörper Nivolumab behandelt wird, er habe zudem kurzzeitig ein Antibiotikum eingenommen, dessen Name er nicht erinnert, es sei wegen eines Harnwegsinfekts gewesen. In der klinisch-neurologischen Untersuchung fallen Ihnen folgende Befunde auf: Hüftabduktion beidseits Kraftgrad 4, Achillessehnen- und Patellarsehnenreflex beidseits erloschen. Wie gehen Sie vor?

○ Stationäre Aufnahme in die Klinik zur elektrophysiologischen Diagnostik, spinale Magnetresonanztomographie und Lumbalpunktion

○ Beim Verdacht auf immuntherapieassoziierte Nebenwirkungen milder Ausprägung Beginn mit 0,5 mg/kg Körpergewicht Prednisolon und Wiedervorstellung in 3 Tagen

○ Vorstellung beim Onkologen des Patienten bei nächster Routinekontrolle

○ Urinstatus und Thoraxröntgen veranlassen

○ Ergänzung einer elektrophysiologischen Diagnostik in Ihrer Praxis am Folgetag

\section{Informationen zur zertifizierten Fortbildung}

Diese Fortbildung wurde von der Ärztekammer Nordrhein für das „Fortbildungszertifikat der Ärztekammer" gemäß $§ 5$ ihrer Fortbildungsordnung mit 3 Punkten (Kategorie D) anerkannt und ist damit auch für andere Ärztekammern anerkennungsfähig.

Anerkennung in Österreich: Für das Diplom-Fortbildungs-Programm (DFP) werden die von deutschen
Landesärztekammern anerkannten Fortbildungspunkte aufgrund der Gleichwertigkeit im gleichen Umfang als DFP-Punkte anerkannt (§ 14, Abschnitt 1, Verordnung über ärztliche Fortbildung, Österreichische Ärztekammer (ÖÄK) 2013).

Hinweise zur Teilnahme:

- Die Teilnahme an dem zertifizierten Kurs ist nur online auf www.springermedizin.de/cme möglich.
- Der Teilnahmezeitraum beträgt 12 Monate. Den Teilnahmeschluss finden Sie online beim Kurs.

- Die Fragen und ihre zugehörigen Antwortmöglichkeiten werden online in zufälliger Reihenfolge zusammengestellt.

- Pro Frage ist jeweils nur eine Antwort zutreffend.

- Für eine erfolgreiche Teilnahme müssen $70 \%$ der Fragen richtig beantwortet werden.

\section{- Teilnehmen können Abonnenten dieser Fachzeitschrift und e.Med- und e.Dent-Abonnenten.}

Gutachter der CME-Fragen der Fortbildungen dieser Zeitschrift: Dr. med. Matthias Kaltenmaier, Heidelberg; Prof. Dr. med. Markus Weih, MME(Bern), Nürnberg 
(3) Eine 48-jährige Patientin mit malignem Melanom unter kombinierter Immuntherapie mit den CheckpointInhibitoren Nivolumab und Ipilimumab präsentiert sich mit Kopfschmerzen, Desorientiertheit und zunehmender Fatigue. Welche Reihenfolge der Diagnostik und Therapie ist sinnvoll?

O Kranielle Magnetresonanztomographie (cMRT) und spinale Magnetresonanztomographie (sMRT), Lumbalpunktion, Beginn Aciclovir und Steroide, Elektroenzephalographie (EEG) und weitere Ausbreitungsdiagnostik

O Direkt mit hochdosierter Steroidtherapie beginnen

O Ausbreitungsdiagnostik für die Tumorerkrankung durchführen, danach cMRT/ sMRT

O Klinische Verlaufskontrolle in 1 Woche, bis dahin Checkpoint-Inhibition pausieren

- Lumbalpunktion und Beginn mit 3-fach antibiotischer Therapie beim Verdacht auf Meningitis nach Leitlinie

? Die kranielle Magnetresonanztomographie (cMRT) bei einem Patienten mit zerebral metastasiertem "nonsmall-cell lung cancer" (NSCLC) zeigt nach Kontrastmittel(KM)-Gabe eine Signalanhebung im Bereich der Nn. faciales. Klinisch-neurologisch besteht eine progrediente Hypakusis, Fatigue, aktuelle tumorspezifische Therapie seit 4 Wochen eine Kombination u.a. mit Pembrolizumab. Zudem hat der Patient eine Ganzhirnbestrahlung vor 8 Wochen erhalten. Bei einer Lumbalpunktion ergibt sich eine mäßige Liquorpleozytose. Welche Differenzialdiagnose ist am wahrscheinlichsten?

O Bakterielle Meningitis

O Toxoplasmose

O Aseptische Meningitis

O Polyneuritis cranialis

O Reizpleozytose nach Ganzhirnbestrahlung
(3) Welche Aussage zur Therapie mit Checkpoint-Inhibitoren ist nicht richtig?

○ Checkpoint-Inhibitoren können bereits nach wenigen Wochen zu therapieassoziierten neurologischen Symptomen führen.

○ Bei der Verdachtsdiagnose eines therapieassoziierten neurologischen Symptoms unter Checkpoint-Inhibition erfolgt umgehend eine Steroidtherapie.

○ Checkpoint-Inhibitoren können zu Hypophysitis führen, dies wurde häufiger unter CTLA4( „cytotoxic T lymphocyte-associated antigen 4")-Blockade (als unter PD1 [,programmed cell death protein 1"]Blockade) beobachtet.

O Checkpoint-Inhibitoren sind bei einigen Tumorentitäten sehr effektiv und führen zu Langzeitüberleben.

O Checkpoint-Inhibitoren können auch zur Behandlung von ZNS (Zentralnervensystem)-Metastasen eigesetzt werden.

3) Was trifft für eine Kombination aus Ganzhirnbestrahlung mit medikamentöser Tumortherapie zu?

O Kann zur schweren und irreversiblen Enzephalopathie führen

○ Darf wegen möglicher Enzephalopathie nicht eingesetzt werden

O Ist bei primären ZNS (Zentralnervensystem)-Malignomen Standardtherapie

O Ist ohne Gefahr neurokognitiver Leistungseinbußen anwendbar

O Kommt nur als Ultima Ratio zum Einsatz 
Hier steht eine Anzeige.

黑 Springer 